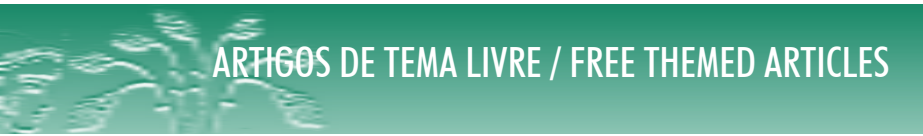

DOI: 10.12957/demetra.2018.32667

\title{
Influência da mídia sobre a aparência de professoras de São Paulo
}

\section{Influence of the media on the appearance of teachers of São Paulo}

\author{
Mariana Gori ${ }^{1}$ \\ Renata Furlan Viebig ${ }^{1,2}$ \\ ${ }^{1}$ Centro Universitário São Camilo. São Paulo, \\ SP, Brasil. \\ ${ }^{2}$ Universidade Presbiteriana Mackenzie. \\ São Paulo, SP, Brasil. \\ Correspondência / Correspondence \\ Mariana Gori \\ E-mail:mariana_gori@hotmail.com
}

\section{Resumo}

Objetivo: Avaliar as influências da mídia sobre a aparência de professoras da Educação Infantil e do Ensino Fundamental I de escolas privadas do município de São Paulo. Metodologia: Estudo transversal realizado com professoras de 4 colégios paulistanos. As docentes foram submetidas a avaliação antropométrica e responderam ao "Questionário de Atitudes Socioculturais" em relação à aparência (SATAQ-3) para verificar a influência da mídia. Resultados: Participaram 55 professoras com idade média de 37,49 anos. A maioria das participantes (76,3\%) foram classificadas nas classes sociais A e B. O Índice de Massa Corporal médio encontrado foi $26,6 \mathrm{Kg} / \mathrm{m}^{2}$ e a média de percentual de gordura corporal foi de 41,9\%. As professoras mais influenciadas pela mídia eram mais velhas, de maior classe socioeconômica e com maior percentual de gordura corporal, porém, somente esta última associação se mostrou estatisticamente significativa. Foi observado que quanto maior o tempo de profissão menor o escore no SATAQ-3 $(\mathrm{p}=0,030)$. Conclusões: As docentes com maior pontuação no questionário tiveram maior peso corporal e percentual de gordura corporal mais elevado. Além disso, as docentes mais experientes sofriam menos influencia da mídia.

Palavras-Chave: Mídia. Influência. Professores. Aparência.

\section{Abstract}

Objective: To evaluate the media's influence on the appearance of female teachers of Early Childhood Education and Primary School I from private schools of São Paulo, SP. Methods: It was a 
cross-sectional study with teachers from 4 schools of São Paulo, SP. The teachers were submitted to an anthropometric assessment and the "Sociocultural Attitude Questionnaire" (SATAQ- 3) was applied to check how media could influence their appearance. Results: Fifty-five teachers, with mean age of 37.49 years old, participated in this study. Most of them (76,3\%) were classified in the socioeconomic classes A and B. The mean Body Mass Index of the teachers was $26.6 \mathrm{Kg} / \mathrm{m}^{2}$ and the mean body fat percentage was $41.9 \%$. Teachers most influenced by the media were older, had higher socioeconomic status and higher percentage of body fat, but only this last association was statistically significant. It was found that teachers with longer experience period presented lower scores in SATAQ $(\mathrm{p}=0.030)$. Conclusions: The teachers with higher scores in the questionnaire had higher body weight and higher body fat percentage. In addition, more experienced teachers were less influenced by the media.

Keywords: Media. Influence. Teachers. Appearance.

\section{Introdução}

O crescente sentimento de desconforto em relação ao corpo e à própria imagem corporal, acompanhados de uma sensação de inadequação, são fenômenos cada vez mais comuns e que têm levado o sujeito contemporâneo, especialmente do gênero feminino, ao adoecimento. ${ }^{1}$

O ideal de corpo passou por mudança substancial em meados do século XX, caracterizandose pela propagação de necessidade de magreza para as mulheres e corpos musculosos para os homens. Embora os padrões de beleza impostos pela sociedade se modifiquem ao longo do tempo, os padrões atuais são onipresentes e estendem-se a toda a comunidade pelos meios de comunicação e redes sociais. ${ }^{2}$

Assim, a influência da mídia é considerada uma das maneiras pelas quais o comportamento de indivíduos e grupos é afetado, sendo que, atualmente, apresenta papel crucial na formação e reflexão da opinião pública, reproduzindo a autoimagem da sociedade. Estudos mostram que a mídia pode influenciar valores, normas e padrões estéticos incorporados pela sociedade moderna, constituindo-se em instrumento transmissor e reforçador dos ideais sociais corporais. ${ }^{3}$

Por outro lado, enquanto o corpo ideal continua a ser promovido pela mídia e pelo discurso social, a necessidade da alimentação rápida e fácil e a publicidade de redes de fast food figuram nos meios de comunicação de forma concomitante e a prevalência de sobrepeso e obesidade aumenta nas sociedades modernas, colocando o indivíduo ainda mais distante do ideal sociocultural. ${ }^{4}$ 
A diferença entre o corpo atual e aquele considerado "ideal" pela mídia pode promover a baixa autoestima, sendo um dos fatores para a insatisfação corporal. ${ }^{5}$ Muitas vezes, tal insatisfação induz comportamentos de risco para Transtornos Alimentares (TAs), como o uso de dietas restritivas e de métodos de controle de peso (uso de laxantes, diuréticos e anabolizantes; indução de vômitos; prática excessiva de atividade física). ${ }^{6,7}$

Gondoli et al. ${ }^{8}$ e Rodgers et al. ${ }^{9}$ salientam que a mídia é o principal agente influenciador negativo na imagem corporal de jovens e crianças. Alguns autores ressaltam que a exposição de corpos de modelos e atrizes costuma causar impacto imediato na idealização morfológica sociocultural, principalmente em sujeitos do gênero feminino. ${ }^{10,11}$

Por outro lado, o papel da escola e de educadores, em especial, aqueles do gênero feminino, como influenciador na promoção da satisfação com a imagem corporal e autoestima de estudantes não tem sido suficientemente investigado. É possível que a insatisfação de professoras com sua própria imagem corporal prejudique seu desempenho no trabalho e influencie negativamente alunos, principalmente os mais jovens, que vislumbram nestas docentes modelos de comportamento.

Neste panorama, o presente estudo buscou avaliar as influências da mídia sobre a satisfação com a imagem corporal de professoras da Educação Infantil e do Ensino Fundamental I de escolas privadas do município de São Paulo.

\section{Métodos}

Trata-se de um estudo transversal, com coleta de dados primários, no qual foram avaliadas professoras da Educação Infantil e Ensino Fundamental I, de 4 colégios particulares da Zona Norte do Município de São Paulo - SP, Brasil.

Como critérios de inclusão, definiu-se que as professoras participantes deveriam ter idade entre 19 e 65 anos e manter contato com os alunos por pelo menos uma vez na semana, sendo incluídas as docentes da educação básica e aquelas que ministravam aulas de inglês, educação física e dança.

\section{Procedimentos}

Inicialmente, todas as professoras voluntárias responderam a um questionário para caracterização socioeconômica e laboral, com ênfase em variáveis relacionadas à formação básica (graduação), disciplina que lecionava, há quanto tempo exercia a profissão e para quais e quantas turmas de alunos ministrava aulas.

Para avaliação da situação socioeconômica foi utilizado o Critério de Classificação Econômica Brasil (CCEB - versão 2016) estabelecido pela Associação Brasileira de Empresas e Pesquisas 
(ABEP), que estima o poder de compra dos brasileiros, classificando a população em estratos econômicos: A1, B1, B2, C1, C2 e D-E. O CCEB está baseado na aquisição dos bens, somando a cada item uma pontuação cujo valor resultante deu a classificação socioeconômica da família segundo os pontos de corte. ${ }^{12}$

Em seguida, as professoras foram submetidas à avaliação do estado nutricional. Para aferição da massa corporal foi utilizada uma balança digital (WISO®) com capacidade para $180 \mathrm{Kg}$ e análise de bioimpedância perna-a-perna, para estimativa do percentual de gordura corporal. As professoras foram pesadas sem calçados e sem peças de vestuário ou objetos pesados.

Para aferição da estatura foi utilizada uma fita métrica inelástica e inextensíveis com capacidade de $2 \mathrm{~m}$, a qual foi fixada verticalmente em uma parede, sem rodapé. As professoras ficaram em pé, em postura ereta, descalços, com os calcanhares juntos e encostados na parede, assim como os ombros e nádegas, mantendo os braços estendidos ao longo do corpo e com os olhos fixos para frente na linha do horizonte (Plano de Frankfurt). ${ }^{13}$ Na sequência, foi colocado um esquadro sobre o vértex do (a) voluntário (a), fixando-a contra a cabeça, para que seja feita a leitura da estatura.

A partir dos valores encontrados (peso e estatura), foi realizado o cálculo do Índice de Massa Corporal (IMC) $\left(\mathrm{kg} / \mathrm{m}^{2}\right)$ mediante a fórmula: $\mathrm{IMC}=\mathrm{P} / \mathrm{E}^{2}$, cujos resultados foram classificados conforme as recomendações da Organização Mundial da Saúde para adultos. ${ }^{14}$

Os valores obtidos de percentual de gordura corporal, por meio da bioimpedância, foram avaliados segundo os pontos de corte propostos por Lohman et al. ${ }^{15}$

\section{Instrumento}

As docentes responderam ao Questionário de Atitudes Socioculturais em relação à aparência (Sociocultural Attitudes Towards Appearance Scale - SATAQ-3) foi desenvolvido por Thompson et al. ${ }^{16}$ traduzido para o português e validado por Amaral et al. ${ }^{10}$ Este instrumento avalia a influência dos aspectos socioculturais na insatisfação corporal de indivíduos. O SATAQ-3 é um questionário auto aplicativo com 30 perguntas, sendo que cada questão apresenta cinco possibilidades de respostas: 1) Discordo totalmente 2) Discordo em grande parte 3) Nem concordo nem discordo 4) Concordo em grande parte 5) Concordo totalmente.

\section{Análise dos dados}

A análise dos dados foi realizada com auxílio do pacote estatístico Statistical Package for the Social Sciences (SPSS) versão 20.0 e o nível de significância adotado foi de $\mathrm{p}<0,05 .{ }^{17}$ Foram utilizados, inicialmente, os recursos da estatística descritiva (média, desvio padrão e frequências). Os dados 
relativos à caracterização da amostra e avaliação do estado nutricional foram descritos em termos de medidas de tendência central e distribuição percentual.

Foi utilizada, para os resultados do SATAQ-3, a análise de variância (ANOVA) para verificar variância homogênea e uma análise de covariância para verificar se a idade, IMC, formação, poderá influenciar nos resultados.

Para verificar a distribuição dos dados foi feito o teste de Kolmogorov-Smirnov. A correlação entre SATAQ com as variáveis (idade, tempo de profissão, peso, estatura, índice de massa corporal e percentual de gordura corporal) foi feita pelo teste de correlação de Pearson. ${ }^{18}$

Foi realizada a análise de variância (ANOVA) com um fator para comparar a pontuação do SATAQ conforme as classificações do IMC e da porcentagem de gordura corporal seguida do post hoc de Bonferroni. Além disso, categorizamos o escore do SATAQ em tercis para compararmos as outras variáveis dependentes. A análise da associação entre a pontuação no SATAQ e as variáveis independentes foram realizadas pela regressão linear multivariada.

\section{Aspectos Éticos}

Todas as professoras preencheram o Termo de Consentimento Livre e Esclarecido (TCLE), após serem esclarecidas sobre os procedimentos do estudo. A pesquisa foi aprovada pelo Comitê de Ética do Centro Universitário São Camilo sob o n.1.709.826.

\section{Resultados}

A amostra foi constituída por 55 docentes, todas do gênero feminino, sendo 49,1\% da Educação Infantil e 50,9\% do Ensino Fundamental I, com idade média de 37,49 anos (DP=12,04).

O tempo médio de exercício da profissão entre as participantes foi de 14,8 anos $(\mathrm{DP}=10,16)$. Grande parte das professoras que participaram da pesquisa eram graduadas em pedagogia, com especialização em psicopedagogia (92,7\%), e ministravam aulas de diversas disciplinas, sendo que $85,5 \%$ das docentes trabalhavam apenas em uma escola (Tabela 1).

Na Tabela 1 é possível observar que 54,5\% das participantes tinha filhos e a maioria das professoras encontravam-se divididas entre as classes sociais A $(32,7 \%)$ e B $(65,4 \%)$.

A Tabela 2 apresenta os resultados relativos à avaliação do estado nutricional das professoras. $\mathrm{O}$ IMC médio encontrado foi de $26,6 \mathrm{Kg} / \mathrm{m}^{2}$, indicativo de sobrepeso. A distribuição em categorias de IMC também apontou que a maioria das professoras $(58,2 \%)$ se encontravam com excesso de peso corporal. O percentual de gordura corporal médio estimado foi de $41,9 \%$, o qual é considerado 
"muito alto" de acordo com os pontos de corte propostos por Lohman. ${ }^{15}$ Apenas 3,6\% das professoras avaliadas apresentaram percentuais de gordura corporal considerados "ideais".

Tabela 1. Distribuição, em números absolutos e percentuais, das variáveis de caracterização socioeconômica das docentes. São Paulo, SP, 2016.

\begin{tabular}{|c|c|}
\hline Variáveis & $f(\%)$ \\
\hline \multicolumn{2}{|l|}{ Idade } \\
\hline $19-29$ & $22(4)$ \\
\hline $30-39$ & $5(9,1)$ \\
\hline $40-49$ & $17(30,9)$ \\
\hline$>50$ & $1(20,0)$ \\
\hline \multicolumn{2}{|l|}{ Escola } \\
\hline 1 & $17(30,9)$ \\
\hline 2 & $14(25,5)$ \\
\hline 3 & $16(29,1)$ \\
\hline 4 & $8(14,5)$ \\
\hline \multicolumn{2}{|l|}{ Formação } \\
\hline Educação Física & $2(3,6)$ \\
\hline Pedagogia / Psicopedagogia & $51(92,7)$ \\
\hline Outros & $2(3,6)$ \\
\hline \multicolumn{2}{|l|}{ Disciplina } \\
\hline Educação física / Dança & $2(3,6)$ \\
\hline Diversas & $50(90,9)$ \\
\hline Outros & $3(5,5)$ \\
\hline \multicolumn{2}{|l|}{ Turma em que ministra aulas } \\
\hline Infantil & $27(49,1)$ \\
\hline Fundamental I & $28(50,9)$ \\
\hline \multicolumn{2}{|l|}{ Trabalha em mais de uma escola } \\
\hline Sim & $8(14,5)$ \\
\hline \multirow[t]{2}{*}{ Não } & $47(85,5)$ \\
\hline & \\
\hline
\end{tabular}




\begin{tabular}{ll}
\hline Variáveis & $\boldsymbol{f}(\%)$ \\
\hline Filhos & \\
Sim & $30(54,5)$ \\
Não & $25(45,5)$ \\
Classificação econômica & \\
A & $18(32,7)$ \\
B1 & $24(43,6)$ \\
B2 & $12(21,8)$ \\
C1 & $1(1,8)$ \\
\hline
\end{tabular}

Tabela 2. Distribuição em números absolutos e percentuais das variáveis antropométricas das docentes participantes. São Paulo, SP, 2016.

\begin{tabular}{lc}
\hline \multicolumn{1}{c}{ Variáveis } & Média $(\mathbf{D P})$ ou $\boldsymbol{f}(\boldsymbol{\%})$ \\
\hline Peso $(\mathrm{kg})$ & $71,0(11,47)$ \\
Estatura $(\mathrm{m})$ & $1,6(0,06)$ \\
IMC $\left(\mathrm{kg} / \mathrm{m}^{2}\right)$ & $26,6(3,81)$ \\
IMC categórico* & \\
Eutrófico & $23(41,8)$ \\
Sobrepeso & $23(41,8)$ \\
Obesidade grau I & $8(14,5)$ \\
Obesidade grau II & $1(1,8)$ \\
$\%$ Gordura corporal & $41,9(7,82)$ \\
$\%$ Gordura corporal categórico* & \\
Ideal & $2(3,6)$ \\
Alto & $10(18,2)$ \\
Moderadamente alto & $1(1,8)$ \\
Muito alto & $42(76,4)$ \\
\hline
\end{tabular}

*dados categóricos. 
A pontuação média das professoras no questionário SATAQ foi de 82,1 pontos $(\mathrm{DP}=18,37)$, sendo que a mais influenciadas atingiram 115,00 pontos e as menos influenciadas marcaram 72,65 pontos.

Conforme os resultados apresentados na Tabela 3, a maioria das professoras menos influenciada pela mídia (Tercil 1) tinham idade entre 40-49 anos, sem que fosse observada diferença estatisticamente significativa. Já as mais influenciadas foram as professoras mais jovens, na faixa etária de 19-29 anos.

As docentes com menores escores no SATAQ eram de classe econômica A e as com maior, de classe B. Além disso, a maior parte das professoras que alcançaram maior pontuação no SATAQ (Tercis 2 e 3) não tinham filhos (40 e 36\%, respectivamente). Entretanto, tais associações não se mostraram estatisticamente significativas.

Tabela 3. Comparação das variáveis de caracterização da profissão (frequência e porcentagem) com a classificação do SATAQ em tercil das participantes. São Paulo, SP, 2016.

\begin{tabular}{lllll}
\hline Variáveis & Tercil 1 & Tercil 2 & Tercil 3 & p-valor \\
\hline Idade & & & & \\
$19-29$ & $4(18,2)$ & $8(36,4)$ & $10(45,5)$ & \\
$30-39$ & $3(60)$ & $1(20)$ & $1(20)$ & 0,442 \\
$40-49$ & $8(47,1)$ & $5(29,4)$ & $4(23,5)$ & \\
$>50$ & $3(27,3)$ & $4(36,4)$ & $4(36,4)$ & \\
Escola & & & & \\
1 & $4(23,5)$ & $6(35,3)$ & $7(41,2)$ & \\
2 & $8(57,1)$ & $3(21,4)$ & $3(21,4)$ & \\
3 & $3(18,8)$ & $8(50)$ & $5(31,2)$ & \\
4 & $3(37,5)$ & $2(25)$ & $3(37,5)$ & \\
Formação & & & & \\
Educação física & $1(50)$ & 0 & $1(50)$ \\
Pedagogia/Psicopedagogia & $16(31,4)$ & $18(35,3)$ & $17(33,3)$ & \\
Outros & $1(50)$ & 0 & $1(50)$ &
\end{tabular}




\begin{tabular}{|c|c|c|c|c|}
\hline Variáveis & Tercil 1 & Tercil 2 & Tercil 3 & p-valor \\
\hline \multicolumn{5}{|l|}{ Disciplina } \\
\hline Educação física/Dança & $1(50)$ & 0 & $1(50)$ & \multirow[t]{3}{*}{0,908} \\
\hline Diversas & $16(32)$ & $17(34)$ & $17(34)$ & \\
\hline Outros & $1(33,3)$ & $1(33,3)$ & $1(33,3)$ & \\
\hline \multicolumn{5}{|l|}{ Sala que ministra aula } \\
\hline Infantil & $7(25,9)$ & $12(44,4)$ & $8(29,6)$ & \multirow[t]{2}{*}{0,300} \\
\hline Fundamental I & $11(39,3)$ & $7(25)$ & $10(35,7)$ & \\
\hline \multicolumn{5}{|c|}{ Se trabalha em mais de uma escola } \\
\hline Sim & $3(37,5)$ & $2(25)$ & $3(37,5)$ & \multirow{2}{*}{0,828} \\
\hline Não & $15(31,9)$ & $17(36,2)$ & $15(31,9)$ & \\
\hline \multicolumn{5}{|l|}{ Filhos } \\
\hline Sim & $12(40)$ & $10(33,3)$ & $8(26,7)$ & \multirow{2}{*}{0,399} \\
\hline Não & $6(24)$ & $9(36)$ & $10(40)$ & \\
\hline \multicolumn{5}{|l|}{ Classificação econômica } \\
\hline $\mathrm{A}$ & $7(38,9)$ & $6(33,3)$ & $5(27,8)$ & \multirow{4}{*}{0,800} \\
\hline $\mathrm{B} 1$ & $7(2,92)$ & $8(33,3)$ & $9(37,5)$ & \\
\hline $\mathrm{B} 2$ & $4(33,3)$ & $5(41,7)$ & $3(25)$ & \\
\hline C1 & 0 & 0 & $1(100)$ & \\
\hline
\end{tabular}

Teste Qui-quadrado; * $\mathrm{p}<0,005$.

Na Tabela 4 observa-se que as docentes integrantes do Tercil 2, conforme pontuação no SATAQ, tinham um peso corporal médio cerca de $9 \mathrm{Kg}$ inferior aos demais tercis $(\mathrm{p}=0,032)$. Foi observada uma tendência de associação entre o IMC médio e a pontuação no SATAQ.

As professoras que foram mais influenciadas pela mídia segundo o SATAQ tinham percentuais de gordura corporal mais elevados $(\mathrm{p}=0,032)$.

A Tabela 5 mostra a análise de correlação entre a pontuação no SATAQ e diversas variáveis do estudo. Observou-se que quanto menor o tempo de profissão das docentes maior era a pontuação no SATAQ, sendo observada correlação inversa e fraca, mas estatisticamente significativa. 
De acordo com a Tabela 6 é possível observar que a medida que aumentava a idade das professoras observava-se uma diminuição de 0,53 pontos no SATAQ ( $\mathrm{p}=0,030)$.

Tabela 4. Análise descritiva (média e desvio padrão e frequência e porcentagem) das variáveis antropométricas com a classificação do SATAQ em tercil das participantes. São Paulo, SP, 2016.

\begin{tabular}{|c|c|c|c|c|}
\hline Variáveis & Tercil 1 & Tercil 2 & Tercil 3 & p-valor \\
\hline Peso (kg) & $74,02(13,02)^{\#}$ & $65,50(10,63)$ & $73,81(8,78)$ & $0,032 *$ \\
\hline Estatura (m) & $1,64(0,05)$ & $1,61(0,06)$ & $1,63(0,05)$ & 0,323 \\
\hline IMC $\left(\mathrm{kg} / \mathrm{m}^{2}\right)$ & $27,25(4,42)$ & $24,92(2,92)$ & $27,71(3,55)$ & 0,054 \\
\hline \multicolumn{5}{|l|}{$\mathrm{IMC}^{\mathrm{a}}$} \\
\hline Eutrófico & $9(39,1)$ & $10(43,5)$ & $4(17,4)$ & \multirow{2}{*}{0,072} \\
\hline Excesso de peso & $9(28,1)$ & $8(25)$ & $15(46,9)$ & \\
\hline$\%$ Gordura corporal & $42,41(7,24)$ & $38,48(7,28)^{\ddagger}$ & $45,10(7,83)$ & $0,032 *$ \\
\hline \multicolumn{5}{|l|}{$\%$ Gordura corporal $^{\mathrm{b}}$} \\
\hline Ideal & 0 & $1(50)$ & $1(50)$ & \multirow{2}{*}{0,603} \\
\hline Acima do ideal & $18(34)$ & $17(32,1)$ & $18(34)$ & \\
\hline
\end{tabular}

Teste Qui-quadrado para dados categóricos; ANOVA com único fator para dados contínuos; Pos hoc de Bonferroni: \# Tercil 1 é diferente de tercil 2; ${ }^{\ddagger}$ Tercil 2 é diferente de tercil 3; p $<0,005$. ${ }^{a}$ Excesso de peso $\left(\mathrm{IMC} \geq 25 \mathrm{Kg} / \mathrm{m}^{2}\right)$;

${ }^{\mathrm{b}}$ Acima do ideal (>35\%).

Tabela 5. Análise de correlação entre pontuação do SATAQ e variáveis das docentes participantes. São Paulo, SP, 2016.

\begin{tabular}{lcl}
\hline Variáveis & $\mathbf{r}$ & p-valor \\
\hline Idade (anos) & $-0,22$ & 0,106 \\
Peso & $-0,02$ & 0,866 \\
Estatura $(\mathrm{m})$ & $-0,03$ & 0,777 \\
IMC $\left(\mathrm{kg} / \mathrm{m}^{2}\right)$ & 0,00 & 0,982 \\
\% Gordura corporal & 0,11 & 0,421 \\
Tempo de profissão & $-0,29$ & $0,030^{*}$ \\
\hline
\end{tabular}

Correlação de Pearson; *p<0,005. 
Tabela 6. Análise de regressão linear univariada para verificar a associação das variáveis independentes sobre SATAQ. São Paulo, SP, 2016.

\begin{tabular}{lccc}
\hline Variáveis & $\boldsymbol{\beta}$ coeficiente & IC95\% & p-valor \\
\hline Idade (anos) & $-0,336$ & $-0,747 ; 0,074$ & 0,106 \\
Tempo de profissão & $-0,529$ & $-1,005 ;-0,053$ & $0,030^{*}$ \\
Peso & $-0,037$ & $-0,478 ; 0,404$ & 0,866 \\
Estatura & $-11,952$ & $-96,192 ; 72,288$ & 0,777 \\
IMC & 0,015 & $-1,312 ; 1,343$ & 0,982 \\
$\%$ Gordura corporal & 0,260 & $-0,383 ; 0,903$ & 0,421 \\
\hline
\end{tabular}

*p<0,005.

\section{Discussão}

Os estudos que investigaram o instrumento SATAQ foram realizados em populações de adultos jovens universitários e adolescentes. Embora, pesquisas envolvendo essa temática tenham chamado maior atenção da comunidade cientifica nos últimos anos, nenhum estudo até o momento aplicou o instrumento para avaliar a influência da mídia em professores, especialmente em âmbito nacional.

A idade média das professoras da presente pesquisa foi de 37,49 anos sendo que 54,5\% tinham filhos e com tempo médio de profissão de 14,8 anos. Uma pesquisa conduzida por Santos \& Marques, ${ }^{21}$ em 59 escolas municipais de Educação Infantil e Ensino Fundamental do Sul do Brasil, mostrou que 70\% dos docentes encontravam-se na classe social B e 18,4\% na classe social A, sendo que quase metade da amostra (47,8\%) tinha até 10 anos de profissão e 68,6\% tinham pelo menos um filho. O perfil dos professores desse estudo se assemelha com o da presente pesquisa.

O IMC médio das docentes do presente estudo foi $26,6 \mathrm{Kg} / \mathrm{m}^{2}$ e o percentual médio de gordura corporal foi 41,9\%. O estudo de Santos \& Marques, ${ }^{21}$ realizado em escolas municipais de Bagé, RS, com 1004 professores, verificou que a prevalência de excesso de peso corporal entre as professoras de Educação Infantil e Ensino Fundamental I foi de 46,7\%. Já uma outra pesquisa, também realizada em uma escola no Sul do país, com professores de Ensino Fundamental, mostrou uma prevalência de $29,4 \%$ de excesso de peso, valores inferiores aos encontrados nos demais estudos. ${ }^{22}$

No presente estudo, mais de metade das professoras apresentaram excesso de peso corporal segundo o IMC $(58,1 \%)$, sendo que quase um quinto da amostra já apresentava obesidade. O estudo de Santos et al., ${ }^{23}$ com professoras de Educação Infantil de uma escola particular de São Paulo, mostrou uma menor prevalência observada em relação ao excesso de peso (52\%), estando $39 \%$ das docentes em sobrepeso e $13 \%$ em obesidade. 
A pontuação média do SATAQ atingida pelas docentes foi de 82,1 pontos, sendo que as professoras com menor pontuação eram mais velhas e com maior tempo de profissão. Além disso, as participantes que marcaram menor pontuação eram de classe socioeconômica mais alta e não tinham filhos.

Nenhum estudo que utilizou esse instrumento para avaliar a influência da mídia em professoras foi encontrado tanto na literatura nacional quanto internacional, o que dificultou a discussão de nossos achados.

Em uma pesquisa brasileira realizada por Alvarenga et al., ${ }^{24} \mathrm{com} 2414$ universitárias de todas as regiões do Brasil, que utilizou o SATAQ como instrumento para avaliar a influência da mídia, mostrou uma pontuação média de 85 pontos, valores acima dos encontrados nesse estudo. A pesquisa mostrou que em relação a faixa etária, as participantes mais velhas apresentavam menor pontuação total, assim como no presente estudo. Em relação a renda, observou-se que as estudantes cuja renda era menor apresentaram menor pontuação no SATAQ, assim como o observado na presente pesquisa.

Um outro estudo nacional desenvolvido por Dunker et al. ${ }^{25}$ com adolescentes entre 15 a 18 anos mostrou que quanto maior a renda familiar maior era pontuação obtida no SATAQ. O escore médio do SATAQ nas adolescentes de escolas públicas foi 86 contra 90 das estudantes de escolas particulares. Esses dados confirmariam a ideia geral de que o ideal de beleza se aplica mais incisivamente a indivíduos de estratos econômicos mais privilegiados. Entretanto, estes resultados não foram verificados em nossa pesquisa.

Um estudo mais recente $(2015)^{26}$ realizado em Juiz de Fora com universitários brasileiros avaliou a prevalência da insatisfação corporal, influência da mídia e comportamento alimentar. A pontuação média do SATAQ entre as mulheres foi 82,7 pontos - valores similares ao do presente estudo. A pesquisa encontrou que quanto maior o IMC e insatisfação corporal, maior e influência recebida da mídia. ${ }^{26}$

Na pesquisa de Felden et al. ${ }^{27}$ com universitários de Santa Catarina que avaliou a internalização dos ideais de corpo mostrou uma pontuação no SATAQ também bem semelhante ao presente estudo, 81,5 pontos para as mulheres. Quando analisadas as subescalas do instrumento, verificou que os homens desejavam ter um corpo mais atlético (musculoso) e as mulheres se sentem mais pressionadas em ter corpos magros/longilíneo pela mídia.

No presente estudo, em relação ao Estado Nutricional, o IMC das participantes que marcaram menor e maior pontuação no SATAQ foi de aproximadamente $27 \mathrm{~kg} / \mathrm{m}^{2}$ sendo que aquelas que obtiveram maior pontuação tinham percentual de gordura corporal médio mais elevado $(45,1 \%)$. No estudo de Alvarenga et al., ${ }^{24}$ da mesma maneira, quanto maior o IMC das participantes maior era a pontuação total no SATAQ. 
Groez et al. ${ }^{28}$ encontraram em sua metanálise, que avaliou 25 estudos internacionais, que o efeito de manipulações experimentais do ideal de beleza pela mídia tem efeito maior em participantes com menor idade. Os pesquisadores ainda observaram uma piora da imagem corporal quando as pessoas estão em contato com imagens de pessoas mais magras. Já Madanat et al., ${ }^{29}$ em sua pesquisa realizada com mulheres na Jordânia, também encontraram que mulheres mais velhas tiveram menores pontuações no SATAQ.

Uma observação atenta das revistas femininas e programas de televisão evidencia como o tema da perda de peso e busca do "corpo ideal" e popular predispõe a uma maior preocupação com o corpo, uso de dietas e comportamentos compensatórios que ajudam a entender que indivíduos mais distantes do ideal sociocultural se sintam piores e mais influenciados e pressionados. ${ }^{30,31}$

Um estudo de avaliação da influência da mídia em jovens adultas na Índia encontrou que a internalização dos ideais da mídia tem papel na insatisfação corporal e busca pela magreza mesmo em um país em desenvolvimento e que, portanto, deve haver fatores de risco potencialmente similares em diferentes culturas. ${ }^{32}$

A identificação de fatores de risco para insatisfação corporal e ocorrência de TAs é essencial para o desenvolvimento e a implantação de programas efetivos de tratamento e prevenção. Um estudo realizado com 173 universitárias americanas demonstrou que a influência da mídia pode ser um fator de risco causal para o início de problemas com a alimentação e o peso e a pressão da mídia influenciaria fortemente as tendências do indivíduo para adotar atitudes e comportamentos alimentares desordenados. ${ }^{33}$

Com relação às limitações do presente estudo, a primeira consideração se refere ao próprio processo de amostragem que, sendo por conveniência e, portanto, não probabilística, devem ser generalizados com cautela. É importante relatar que houve dificuldade em conseguir que outras instituições de ensino permitissem a realização deste estudo com seus docentes, para que pudéssemos ampliar a amostra desta pesquisa.

Além disso, nosso estudo foi limitado apenas à análise de dados de professoras de Educação Infantil e Ensino Fundamental I, portanto, é interessante que seja replicado com um número maior de amostra e mais diversificada, em escolas públicas e em outras cidades e regiões brasileiras.

O instrumento utilizado para verificar a influência recebida pela mídia SATAQ-3 não possui classificação própria, impossibilitando afirmar se os professores apresentam baixa ou alta influência midiática. 


\section{Conclusões}

A pontuação média obtida no SATAQ pelas docentes do presente estudo foi menor do que a relatada nos escassos estudos realizados no país e no mundo. Entretanto, nenhuma destas pesquisas foi realizada com professores.

Houve correlação inversa e significativa entre o tempo de exercício da docência e pontuação no SATAQ-3, mostrando que docentes mais experientes sofriam menos influencia da mídia. Além disso, as docentes com maior pontuação no questionário tiveram maior peso corporal e percentual de gordura mais elevado.

É importante que docentes que trabalham com crianças ainda em idade precoce e em fase formativa da personalidade, as quais são consideradas modelos para seus alunos, ampliem seus conhecimentos sobre a influência da mídia e seu papel nos TAs e na imagem corporal, para que possam incorporar esses temas em seus programas educativos em sala de aula.

\section{Colaboradores}

Gori M realizou o levantamento bibliográfico, coleta de dados e participou da análise e interpretação destes. Renata Viebig RF foi responsável pela concepção do estudo como orientadora do trabalho. Participou efetivamente na análise e interpretação dos dados.

Conflito de interesses: Os autores declaram não haver conflito de interesses.

\section{Referências}

1. Novaes JV, Iannelli AM. A dimensão simbólica do corpo e o fenômeno social da corpolatria. Revista Interdisciplinar da Universidade Federal do Tocantins. 2015; 2(1):176-189.

2. Neighbors LA, Neighbors J. Prevalence and magnitude of body weight and shape dissatisfaction among university students. Eating Behavior. 2007; 8(4):429-439.

3. Ditamar H. Vulnerability factors and processes linking sociocultural pressures and body dissatisfaction. J Soc Clin Psychol. 2005; 24(1):1081-1087.

4. Ogden CL, Carroll MD, Curtin LR, McDowell MA, Tabak CJ, Flegal KM. Prevalence of overweight and obesity in the United States, 1999-2004. JAMA. 2006; 295(13):1549-1555.

5. Santos VM, Mezzaroba C. A percepção da imagem corporal: algumas representações de corpo na juventude. EFDeportes Revista Digital. 2013; 18(32):170-181.

6. Alvarenga M, Dunker KLL. Padrão e comportamento alimentar na anorexia e na bulimia nervosa. In: Philippi ST, Alvarenga M. Transtornos alimentares: uma visão nutricional. São Paulo: Manole; 2004. 
7. Hunt TJ, Thienhaus E, Elwood A. The mirror lies: body dysmorphic disorder. American Family Physician. 2008; 78(2):217-222.

8. Gondoli DM, Corning AF, Salafia EH, Bucchianeri MM, Fitzsimmons EE. Heterosocial involvement, peer pressure for thinness, and body dissatisfaction among young adolescent girls. Body Image. 2011; 8(2):143-148.

9. Rodgers R, Chabrol H, Paxton SJ. An exploration of the tripartite influence model of body dissatisfaction and disordered eating among Australian and French college women. Body image. 2011; 8(1):208-215.

10. Amaral ACS, Cordás TA, Conti MA, Ferreira MEC. Equivalência semântica e avaliação da consistência interna da versão em português do Sociocultural Attitudes Towards Appearance Questionnaire-3 (SATAQ-3). Cad Saúde Pública. 2011; 27(8):1487-1497.

11. Flament MF, Hill EM, Buchholz A, Henderson K, Tasca GA, Goldfield G. Internalization of the thin and muscular body ideal and disordered eating in adolescence: The mediation effects of body esteem. Body Image. 2012; 9(1):68-75.

12. Critério Brasil. Classificação Econômica Brasil. ABEP - Associação Brasileira de Empresas de Pesquisa [Internet]. [acesso em: 21 jun. 2016]. Disponível em: http://www.abep.org/criterio-brasil

13. Nassif MAL, Viebig RF. Avaliação antropométrica no ciclo da vida: uma visão prática. São Paulo: Metha; 2011.

14. World Health Organization. WHO Obesity: preventing and managing the global epidemic. Report of a WHO Consultation on Obesity. Geneve: WHO; 1995.

15. Lohmann RA. The commons: new perspectives on nonprofit organizations and voluntary action. ResearchGate [Internet] Jan. 1992. Disponível em: https://www.researchgate.net/ publication/258844913_The_Commons_New_Perspectives_on_Nonprofit_Organization_ Voluntary_Action_and_Philanthropy

16. Thompson JK, van den Berg P, Roehrig M, Guarda AS, Heinberg LJ. The sociocultural atitudes towards apperance scale-3 (SATAQ-3): development and validation. Int J Eat Disord. 2004; 35(1):294-304.

17. Norusis MJ. SPSS for Windows: Advanced Statistics, release 6.0. Chicago: SPSS Inc., 1993.

18. Field A. Descobrindo a estatística usando o SPSS. Porto Alegre: Artmed; 2009.

19. Kachani AT. Checagem do corpo em transtornos alimentares: relação entre comportamentos e cognições. 2012. 210f. Tese [Doutorado]. [São Paulo]: Programa de Pós-Graduação em Fisiopatologia Experimental, Faculdade de Saúde Pública; 2012.

20. Alves T, Pinto JMR. Remuneração e características do trabalho docente no brasil: um aporte. Cad Pesquisa. 2011; 41(13):110-121.

21. Santos MN, Marques AC. Condições de saúde, estilo de vida e características de trabalho de professores de uma cidade do sul do Brasil. Ciênc Saúde Coletiva. 2013; 18(3):837-846.

22. Prass PBB, Oliveira WC. A Interface da autoavaliação em saúde dos professores e seu preparo para abordagem do tema na escola. Revista Brasileira de Ciências da Saúde. 2015; 19(2):45-52. 
23. Santos DLC, Cassimiro TC, Simony RF, Valente MLF, Olim SCV, Marum RH. Avaliação nutricional e estilo de vida de educadoras de uma escola do município de São Paulo. O Mundo da Saúde. 2011; 35(4):454-458.

24. Alvarenga MS, Dunker KLL, Philippi ST, Scagliusi FB. Influência da mídia em universitárias brasileiras de diferentes regiões. J Bras Psiquiatr. 2010; 59(2):111-118.

25. Dunker KLL, Fernandes CPB, Carreira Filho D. Influência do nível socioeconômico sobre comportamentos de risco para transtornos alimentares em adolescentes. J Bras Psiquiatr. 2009; 58(3):156-161.

26. Batista A, Neves CM, Meireles JFF, Ferreira MEC. Dimensão atitudinal da imagem corporal e comportamento alimentar em graduandos de educação física, nutrição e estética da cidade de Juiz de Fora - MG. Rev Educ Fís. UEM. 2015; 26(1):69-77.

27. Felden EPG, Pio IG, Santos MO, Barbosa DG, Andrade RD, Pelegrini A. Internalização dos ideais de corpo em acadêmicos de Educação Física e Fisioterapia. Revista Brasileira de Ciência e Movimento. 2016; 24(4):121-128.

28. Groez LM, Levine MP, Murnen SK. The effect of experimental presentation of thin mediaimages on body satisfaction: a meta-analytic review. Int J Eat Disord. 2002; 31(1):1-16.

29. Madanat HD, Hawks SR, Brown RB. Validation of the sociocultural attitudes towards appearance questionnaire-3 among a random sample of Jordanian women. Body Image. 2006; 3(1):421-425.

30. Martinez-Gonzalez MA, Gual P, Lahortiga F, Alonso Y, Irala-Estévez J, Cervera S. Parentafactors, mass media influences, and the onset of eating disorders in a prospective population- based cohort. Pediatrics. 2003; 111(1):315-320.

31. Serra GMA, Santos LM. Saúde e mídia na construção da obesidade e corpo perfeito. Ciênc Saúde Coletiva. 2003; 8(2):691-701.

32. Shroff H, Thompson JK. Body image and eating disturbance in India: media and interpersonal influences. Int J Eat Disord. 2004; 35(2):198-203.

33. Thompson JK, Coovert MD, Stormer SM. Body image, social comparison, and eating disturbance: A covariance structure modeling investigation. Int J Eat Disord. 1999; 26(1):43-51.

34. Wilksch SM, Tiggemann M, Wade TD. Impact of interactive school-based media literacy lessons for reducing internalization of media ideals in young adolescent girls and boys. Int J Eat Disord. 2006; 39(1):385-393.

Recebido: 02 de fevereiro de 2018

Revisado: 22 de maio de 2018

Aceito: 15 de junho de 2018 